\title{
Development of ten polymorphic microsatellite loci for the sea snake Hydrophis elegans (Elapidae: Hydrophiinae) and cross-species amplification for fifteen marine hydrophiine species
}

\author{
Vimoksalehi Lukoschek • John C. Avise
}

Received: 5 January 2011/Accepted: 17 January 2011/Published online: 30 January 2011

(C) The Author(s) 2011. This article is published with open access at Springerlink.com

\begin{abstract}
We developed ten microsatellite loci for the elegant sea snake, Hydrophis elegans, from partial genomic DNA libraries using a repeat enrichment protocol. Eight loci had nine or more alleles per locus (maximum 20 ), while the other two had three and seven. All ten loci amplified successfully in 11 of the 15 additional hydrophiine sea snake species screened. Nine loci amplified successfully for three species and eight amplified successfully for the remaining species. Based on this highly successful cross-amplification we expect these ten loci to be useful markers for investigating population genetic structure, gene flow and parentage for all sea snake species from the Hydrophis group.
\end{abstract}

Keywords Microsatellite loci - Hydrophiinae ·

Hydrophis · Sea snakes · Connectivity · Parentage

Recently published IUCN Red List Assessments for all true sea snake species (Elapidae: Hydrophiinae) listed four of 54 species in Threatened or Near Threatened categories and 21 species as Data Deficient (IUCN 2010). Indeed, many aspects of the ecology and biology of sea snakes remain poorly understood and difficulties of direct observation in marine systems hinder significant progress. Highresolution molecular markers, such as nuclear microsatellites, provide compelling alternatives for addressing critical

V. Lukoschek · J. C. Avise

Department of Ecology and Evolutionary Biology,

University of California at Irvine, Irvine, CA 92697, USA

V. Lukoschek $(\bowtie)$

ARC Centre of Excellence for Coral Reef Studies,

James Cook University, Townsville, QLD 4811, Australia

e-mail: vimoksalehi.lukoschek@jcu.edu.au questions about population genetic structure, gene flow, dispersal, effective population sizes and mating systems. Microsatellite loci have only been developed for one sea snake species, Aipysurus laevis, (Lukoschek et al. 2005) and large-scale genotyping revealed relatively low polymorphism at most loci (Lukoschek et al. 2008). Moreover, true sea snakes comprise two evolutionary lineages (Lukoschek and Keogh 2006), the Aipysurus and Hydrophis groups, and microsatellites developed for A. laevis do not amplify in Hydrophis group species (Lukoschek 2008). The 39 Hydrophis group species are closely related (Lukoschek and Keogh 2006), so in order to obtain polymorphic markers for this group we developed microsatellite loci for Hydrophis elegans and conducted cross-species amplification trials for 13 Hydrophis group species.

We employed a modified version of a hybridization capture protocol using magnetic streptavidin beads and biotinylated probes (Hamilton et al. 1999; Hauswaldt and Glenn 2003) to enrich for microsatellites in a genomic library for H. elegans. Our protocol followed Mackiewicz et al. (2006) with one exception: we used a cocktail of biotinylated repeat probes comprising $(\mathrm{TG})_{12},(\mathrm{AG})_{12}$, $(\mathrm{ATC})_{8},(\mathrm{AAC})_{8},(\mathrm{ACAG})_{6},(\mathrm{ACTG})_{6}$, and $(\mathrm{AGAT})_{7}$. A total of 129 inserts were sequenced and microsatellite repeat regions detected by eye. Primers pairs were designed for all 24 inserts containing microsatellites using OligoAnalyzer 3.0 (Integrated DNA Technologies) and tested on H. elegans $(\mathrm{n}=24)$. One primer from each pair was $5^{\prime}$ end labeled with a tag (5'-GGAAACAGCTATGACCATG$3^{\prime}$ ) for tailed PCR with an M13 primer labeled with a 6-FAM, HEX, or NED (Applied Biosystems) fluorophore. A subset of ten microsatellite loci amplified consistently and without multiple peaks. These were screened further in $H$. elegans and used in cross-species amplifications (Table 1). 
Table 1 Characteristics of ten microsatellite loci developed for Hydrophis elegans

\begin{tabular}{|c|c|c|c|c|c|}
\hline Locus & Repeat motif & Primer sequence $\left(5^{\prime}-3^{\prime}\right)(\mathrm{F} / \mathrm{R})$ & $\begin{array}{l}\mathrm{Tm} \\
\left({ }^{\circ} \mathrm{C}\right)\end{array}$ & $\begin{array}{l}\text { Expected } \\
\text { product } \\
\text { size (bp) }\end{array}$ & $\begin{array}{l}\text { GenBank } \\
\text { accession } \\
\text { no. }\end{array}$ \\
\hline $\mathrm{He} 792$ & $(\mathrm{AC})_{3}(\mathrm{TCAC})_{2}(\mathrm{TC})_{15}(\mathrm{AC})_{9}$ & $\begin{array}{l}\text { M13-ATTGGAGCAGCTCTGAAGGACTGT } \\
\text { CGTTTCTCCTTGGCCTGGATGATT }\end{array}$ & 52 & 143 & JF261162 \\
\hline $\mathrm{He} 730$ & $(\mathrm{GA})_{18}$ & $\begin{array}{l}\text { M13-GAGTGTTTGTGGCTGAACCAGTTTG } \\
\text { TGAGACACCTCACAAGGG }\end{array}$ & 52 & 179 & JF261163 \\
\hline He919 & $(\text { TAGA })_{4}$ TAGG(TAGA) ${ }_{8}$ AAGA(TAGA $)_{3}$ & $\begin{array}{l}\text { M13-GGACTTCCTGCTCAGTACTTGTGT } \\
\text { GCATTGGCTAGAGCAAGTGCATGT }\end{array}$ & 52 & 217 & JF261164 \\
\hline He967 & $(\mathrm{GT})_{3} \mathrm{AT}(\mathrm{GT})_{9}(\mathrm{GA})_{19}$ & $\begin{array}{l}\text { M13-ATCCTTCCTACCAGCCACAACCAT } \\
\text { CAGGTTGTTGTTGATCCTTGGTGA }\end{array}$ & 55 & 227 & JF261165 \\
\hline He953 & $\left.(\mathrm{TG})_{10} \mathrm{TTTGTA} \mathrm{TG}\right)_{8}(\mathrm{AG})_{7}$ & $\begin{array}{l}\text { M13-GCTCTGACAATACATGGATGGCGT } \\
\text { GGCGACTTTAAGGCAGCATAGGTT }\end{array}$ & 55 & 268 & JF261166 \\
\hline $\mathrm{He} 778$ & $(\mathrm{AG})_{8} \mathrm{GTAT}(\mathrm{GA})_{6} \mathrm{CA}(\mathrm{GA})_{5} \mathrm{CAGAGG}(\mathrm{GA})_{4} \mathrm{AA}(\mathrm{GA})_{6}$ & $\begin{array}{l}\text { M13-AAGGAAGGAGACAGAAGCGAACCA } \\
\text { CACCTGGGAATTCTAGGATCAAGC }\end{array}$ & 55 & 177 & JF261167 \\
\hline He793 & $(\mathrm{GATA})_{9} \mathrm{~A}(\mathrm{ATAG})_{10}$ & $\begin{array}{l}\text { M13-GTGGTCTTGACACAACTTGAATGC } \\
\text { CCAGCATTAGGAATCTGATGAAGGGAGC }\end{array}$ & 55 & 227 & JF261168 \\
\hline He978 & $(\mathrm{AC})_{5} \mathrm{AAAC}(\mathrm{AG})_{9}(\mathrm{ACAG})_{3}(\mathrm{ACAGAG})_{3}$ & $\begin{array}{l}\text { M13-GGGCTTCATCATAAAGGTCACAATGC } \\
\text { CGCAGAAGTAGGATCAATGGTAGC }\end{array}$ & 55 & 268 & JF261169 \\
\hline $\mathrm{He} 962$ & $(\mathrm{CT})_{11} \mathrm{CATT}(\mathrm{CT})_{7} \mathrm{TTAT}(\mathrm{GT})_{4}$ & $\begin{array}{l}\text { M13-TGAGCTTCAAGGGAGCTGACCATA } \\
\text { GGTGCATTAGACTCATCAAGAGTACCAC }\end{array}$ & 55 & 254 & JF261170 \\
\hline He706 & $(\mathrm{GT})_{8}(\mathrm{GA})_{12}(\mathrm{GGGA})_{4} \mathrm{AGGG}(\mathrm{GA})_{5} \mathrm{CA}(\mathrm{GA})_{2}(\mathrm{CAGA})_{5}$ & $\begin{array}{l}\text { M13-GGGTGAAGCATCTGATAGTCTGTG } \\
\text { AGTCACTGTACGAGGCAGTTGTGA }\end{array}$ & 52 & 335 & JF261171 \\
\hline
\end{tabular}

Amplifications of microsatellite loci were performed in a $10 \mu \mathrm{l}$ volume containing $1 \times$ GoTaq reaction buffer (which included $1.5 \mathrm{mM} \mathrm{MgCl}_{2}$ ), $0.25 \mu \mathrm{g}$ bovine serum albumin, $0.2 \mathrm{mM}$ each dNTP, $0.25 \mu \mathrm{M}$ M13-labeled primer, $0.25 \mu \mathrm{M}$ locus-specific primer, $0.025 \mu \mathrm{M}$ tailed locus-specific primer, $0.4 \mathrm{U}$ GoTaq DNA polymerase (Promega), and $1 \mu \mathrm{l}$ genomic DNA. Amplifications were conducted using an initial denaturation step at $95^{\circ} \mathrm{C}$ for 5 min, followed by 32 cycles of $95^{\circ} \mathrm{C}$ for $40 \mathrm{~s}$, locus specific annealing temperatures (Tm in Table 1) for $40 \mathrm{~s}$, and $72^{\circ} \mathrm{C}$ for $1 \mathrm{~min}$. PCR products were pooled into two groups, diluted ten-fold and electrophoresed on an ABI 3130xl automatic sequencer. Alleles were sized using a ROX labeled GS500 internal standard and scored using GeneMapper 4.0 (Applied Biosystems).

We screened 71 adult $H$. elegans from three regions around Australia. Cross-species amplifications were conducted for 13 Hydrophis group species plus two 'primitive' species (Lukoschek and Keogh 2006). For one species, Lapemis curtus, we screened 76 individuals while sample sizes for the remaining 14 species ranged from 1 to 14 (Table 2), typically from one or two locations per species. Samples were mostly obtained from trawler by-catch but also museum collections. Genotypic frequencies for species with $N \geq 10$ were tested for conformance to HardyWeinberg equilibrium (HWE) using the exact test (Guo and Thompson 1992) while linkage disequilibrium (LD) was tested in $H$. elegans and $L$. curtus using the exact test implemented in GenePop Web Version 4.0.10 (Raymond and Rousset 1995; Rousset 2008).

For $H$. elegans, nine microsatellite loci had moderate to high numbers of alleles (7-20) per locus and six had expected heterozygosities $\left(H_{e}\right) \geq 0.80$ (Table 2). Genotype frequencies at four loci departed from Hardy-Weinberg equilibrium (HWE) at $P<0.05$ (Table 2), but none remained significant after Bonferroni correction. Two pairs of loci were in linkage disequilibrium (LD) at $P<0.05$ but only one pair (He978 and He706) remained significant after Bonferroni correction. This locus-pair was not in LD for L. curtus (see below), suggesting a sampling effect rather than physical genetic linkage. For Lapemis curtus, numbers of alleles per locus ranged from 3 to 16 with three loci having more alleles than for $H$. elegans (Table 2). However, expected heterozygosities in L. curtus typically were lower than in $H$. elegans, with eight loci having $H_{e}<0.80$ (Table 2). Genotype frequencies at two loci departed from HWE at $P<0.05$ (Table 2) but none remained significant after Bonferroni correction. Six pairs of loci were in LD at $P<0.05$ but did not include He978 and $\mathrm{He} 706$, and none remained significant after Bonferroni correction.

All ten loci amplified successfully in ten of the 14 additional sea snake species screened while nine loci amplified successfully for three species and eight for the 
Table 2 Attributes of ten microsatellite loci developed for Hydrophis elegans and the results of cross-species amplification trials for 15 hydrophiine sea snake species

\begin{tabular}{|c|c|c|c|c|c|c|c|c|c|c|}
\hline \multirow[t]{2}{*}{ Locus } & \multicolumn{5}{|c|}{ Hydrophis elegans $(\mathrm{N}=71)$} & \multicolumn{5}{|c|}{ Lapemis curtus $(\mathrm{N}=76)$} \\
\hline & Size range (bp) & $\mathrm{N}$ & $\mathrm{Na}$ & Ho & $\mathrm{He}$ & Size range (bp) & $\mathrm{N}$ & $\mathrm{Na}$ & Ho & $\mathrm{He}$ \\
\hline He792 & $148-180$ & 69 & 14 & 0.77 & 0.86 & $142-162$ & 75 & 8 & 0.40 & 0.43 \\
\hline $\mathrm{He} 730$ & $188-230$ & 61 & 20 & 0.77 & 0.80 & 184-208 & 73 & 6 & 0.68 & 0.62 \\
\hline He919 & $212-248$ & 60 & 9 & 0.83 & 0.80 & $192-276$ & 73 & 16 & 0.86 & 0.88 \\
\hline He967 & $225-267$ & 41 & 18 & $0.76^{*}$ & 0.90 & $215-251$ & 67 & 8 & $0.57 *$ & 0.67 \\
\hline He953 & $270-298$ & 65 & 11 & 0.82 & 0.81 & $282-296$ & 73 & 3 & 0.08 & 0.08 \\
\hline $\mathrm{He} 778$ & 194, 196, 198 & 68 & 3 & 0.24 & 0.23 & $188-212$ & 76 & 4 & 0.50 & 0.47 \\
\hline He793 & $204-248$ & 67 & 12 & 0.85 & 0.85 & $210-268$ & 71 & 13 & $0.85^{*}$ & 0.87 \\
\hline He978 & $270-290$ & 60 & 7 & $0.62 *$ & 0.66 & $270-292$ & 72 & 3 & 0.07 & 0.07 \\
\hline $\mathrm{He} 962$ & $266-298$ & 69 & 10 & $0.26^{*}$ & 0.41 & $272-300$ & 73 & 6 & 0.34 & 0.34 \\
\hline $\mathrm{He} 706$ & $344-360$ & 63 & 9 & $0.71 *$ & 0.75 & $346-362$ & 54 & 4 & 0.09 & 0.09 \\
\hline \multirow[t]{2}{*}{ Locus } & \multicolumn{6}{|c|}{ Hydrophis occellatus $(\mathrm{N}=14)$} & \multicolumn{4}{|c|}{ Astrotia stokesii $(\mathrm{N}=2)$} \\
\hline & \multicolumn{2}{|l|}{ Size range $(b p)$} & $\mathrm{N}$ & $\mathrm{Na}$ & Ho & $\mathrm{He}$ & \multicolumn{2}{|l|}{ Size range (bp) } & $\mathrm{N}$ & $\mathrm{Na}$ \\
\hline $\mathrm{He} 792$ & \multicolumn{2}{|l|}{$156-164$} & 14 & 4 & 0.43 & 0.53 & 148,152 & & 2 & 2 \\
\hline He730 & 196-218 & & 13 & 9 & 0.92 & 0.85 & 194 & & 2 & 1 \\
\hline He919 & $220-244$ & & 12 & 7 & 0.83 & 0.81 & 232 & & 1 & 1 \\
\hline He967 & $233-245$ & & 14 & 6 & 0.64 & 0.69 & N/A & & 0 & N/A \\
\hline He953 & $270-286$ & & 13 & 7 & 0.85 & 0.79 & 288 & & 2 & 1 \\
\hline $\mathrm{He} 778$ & $162-210$ & & 12 & 6 & 0.67 & 0.70 & 186,196 & & 2 & 2 \\
\hline He793 & $220-248$ & & 13 & 8 & 0.77 & 0.76 & $214-242$ & & 2 & 4 \\
\hline He978 & 278,286 & & 11 & 2 & 0.18 & 0.17 & 280 & & 2 & 1 \\
\hline He962 & $282,288,290$ & & 14 & 3 & $0.43 *$ & 0.61 & 286,290 & & 2 & 2 \\
\hline $\mathrm{He} 706$ & 344 & & 10 & 1 & N/A & N/A & $348,358,370$ & & 2 & 3 \\
\hline Locus & Hydrophis lapemoi & $(\mathrm{N}$ & $=11)$ & & & Hydrophis macd & dowelli $(\mathrm{N}=10$ & & & \\
\hline & Size range (bp) & $\mathrm{N}$ & $\mathrm{Na}$ & Ho & $\mathrm{He}$ & Size range (bp) & $\mathrm{N}$ & $\mathrm{Na}$ & Но & $\mathrm{He}$ \\
\hline He792 & $142-156$ & 11 & 6 & 0.82 & 0.69 & $140-150$ & 9 & 2 & 0.56 & 0.40 \\
\hline $\mathrm{He} 730$ & 192-204 & 11 & 4 & $0.18 *$ & 0.58 & $184,194,204$ & 10 & 3 & 0.90 & 0.62 \\
\hline He919 & $212-240$ & 11 & 7 & 0.73 & 0.79 & $236-256$ & 7 & 6 & 0.71 & 0.76 \\
\hline He967 & $231-249$ & 10 & 6 & 0.80 & 0.75 & $229-241$ & 9 & 4 & 0.33 & 0.38 \\
\hline He953 & $286-296$ & 10 & 5 & 0.60 & 0.63 & $272-292$ & 9 & 5 & 0.67 & 0.64 \\
\hline $\mathrm{He} 778$ & $188,190,196$ & 9 & 3 & 0.22 & 0.20 & 186,198 & 9 & 2 & 0.56 & 0.48 \\
\hline He793 & $214-230$ & 11 & 5 & 0.82 & 0.78 & $224-260$ & 10 & 7 & 1.00 & 0.83 \\
\hline He978 & 278 & 11 & 1 & N/A & N/A & $286,290,294$ & 9 & 3 & 0.78 & 0.55 \\
\hline $\mathrm{He} 962$ & $282-292$ & 10 & 4 & 0.30 & 0.35 & 270,272 & 10 & 2 & 0.10 & 0.10 \\
\hline He706 & $348-354$ & 11 & 4 & $0.27 *$ & 0.43 & 334 & 10 & 1 & N/A & N/A \\
\hline Locus & Pelamis platurus & $\mathrm{N}=$ & & & & & Hydrophis brook & $i(\mathrm{~N}=$ & & \\
\hline & Size range (bp) & & $\mathrm{N}$ & $\mathrm{Na}$ & Ho & $\mathrm{He}$ & Size range (bp) & & $\mathrm{N}$ & $\mathrm{Na}$ \\
\hline $\mathrm{He} 792$ & $146-156$ & & 10 & 5 & 0.70 & 0.74 & 154 & & 1 & 1 \\
\hline He730 & $182-200$ & & 10 & 6 & 0.60 & 0.64 & N/A & & 0 & 0 \\
\hline He919 & $216-240$ & & 5 & 4 & 0.80 & 0.70 & 224,228 & & 1 & 2 \\
\hline He967 & $227-245$ & & 7 & 7 & 0.86 & 0.76 & 247,249 & & 1 & 2 \\
\hline He953 & 284-294 & & 9 & 6 & $0.44 *$ & 0.78 & 288, 292 & & 1 & 2 \\
\hline $\mathrm{He} 778$ & 176-198 & & 10 & 4 & 0.30 & 0.27 & 208 & & 1 & 2 \\
\hline
\end{tabular}


Table 2 continued

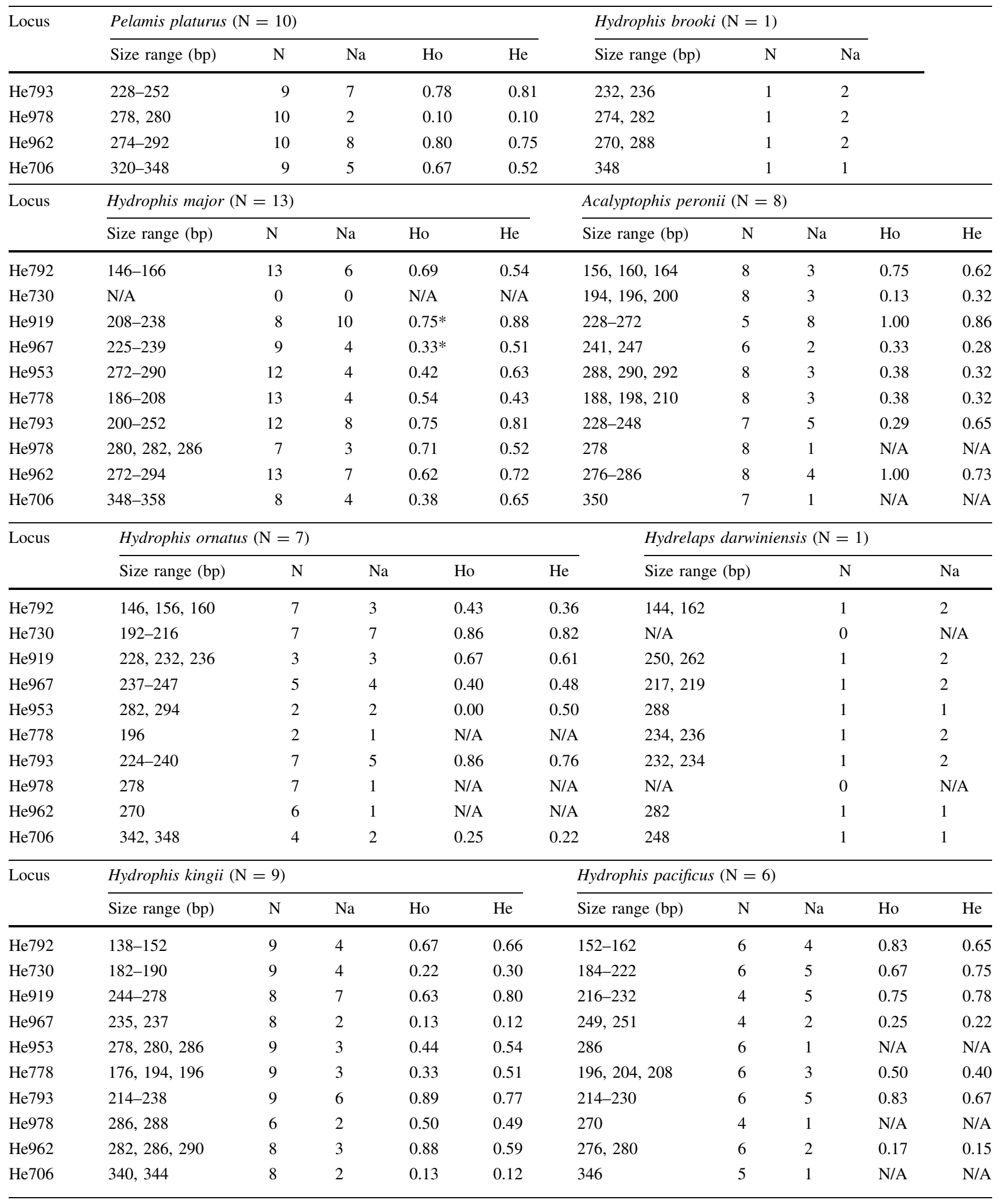


Table 2 continued

\begin{tabular}{|c|c|c|c|c|c|c|c|c|}
\hline \multirow[t]{2}{*}{ Locus } & \multicolumn{5}{|c|}{ Hydrophis cyanocinctus $(\mathrm{N}=3)$} & \multicolumn{3}{|c|}{ Parahydrophis mertoni $(\mathrm{N}=1)$} \\
\hline & Size range (bp) & $\mathrm{N}$ & $\mathrm{Na}$ & Ho & $\mathrm{He}$ & Size range $(b p)$ & $\mathrm{N}$ & $\mathrm{Na}$ \\
\hline $\mathrm{He} 792$ & $144-150$ & 3 & 3 & 0.33 & 0.50 & 154 & 1 & 1 \\
\hline He730 & 190,194 & 3 & 2 & 0.33 & 0.28 & 184 & 1 & 1 \\
\hline He919 & $216-232$ & 2 & 3 & 1.00 & 0.63 & 228 & 1 & 1 \\
\hline He967 & $235-251$ & 3 & 4 & 0.67 & 0.67 & N/A & 0 & N/A \\
\hline He953 & 286,288 & 3 & 2 & 0.67 & 0.44 & 284,294 & 1 & 2 \\
\hline $\mathrm{He} 778$ & 192,208 & 3 & 2 & 0.33 & 0.50 & 196 & 1 & 1 \\
\hline He793 & $218-234$ & 3 & 5 & 1.00 & 0.78 & 226,230 & 1 & 2 \\
\hline He978 & 294 & 1 & 1 & N/A & N/A & 282 & 1 & 1 \\
\hline He962 & 276,278 & 3 & 2 & 0.33 & 0.28 & 294 & 1 & 1 \\
\hline He706 & 342,348 & 3 & 2 & 0.33 & 0.28 & 356 & 1 & 1 \\
\hline
\end{tabular}

$\mathrm{N}$ is the number of samples that successfully amplified and were scored for each locus. $\mathrm{Na}$ is the number of alleles. $\mathrm{Ho}$ and $\mathrm{He}$ refers to observed and expected heterozygosity calculated by GenAlEx (Peakall and Smouse 2006). Hardy-Weinberg Equilibrium was evaluated for the seven species with sample sizes of ten or more snakes

Loci that deviated from HWE are indicated by $*(P=0.05)$

remaining species (Table 2). Allele sizes and frequency distributions varied considerably among species (Table 2). A few loci departed from HWE for the five species with $N \geq 10$ (Table 2) but different loci typically were involved suggesting sampling artefacts. Only two of 225 tests showed departures from $\operatorname{LD}(P<0.05)$ and none remained significant after Bonferroni correction. These highly successful cross-amplifications indicate that these ten loci will be useful for investigating population genetic structure, gene flow and parentage for all Hydrophis group species, plus the three 'primitive' Australian endemics.

Acknowledgments This work was supported by the Sea World Research and Rescue Foundation; the University of California, Irvine; and the ARC Centre of Excellence for Coral Reef Studies. We thank Andrei Tatarenkov, Felipe Barreto and Rosemary Byrne for lively discussions about microsatellite development.

Open Access This article is distributed under the terms of the Creative Commons Attribution Noncommercial License which permits any noncommercial use, distribution, and reproduction in any medium, provided the original author(s) and source are credited.

\section{References}

Guo SW, Thompson EA (1992) Performing the exact test of HardyWeinberg proportion for multiple alleles. Biometrics 48:361-372

Hamilton MB, Pincus EL, Di Fiore A, Flescher RC (1999) Universal linker and ligation procedures for construction of genomic DNA libraries enriched for microsatellites. BioTechniques 27:500-507
Hauswaldt JS, Glenn TC (2003) Microsatellite DNA loci from the diamondback terrapin (Malaclemys terrapin). Mol Ecol Notes 3:174-176

IUCN (2010) IUCN red list of threatened species. Version 2010.4. www.iucnredlist.org

Lukoschek V (2008) Molecular ecology, evolution and conservation of hydrophiine sea snakes. Ph.D. Thesis, James Cook University, Townsville

Lukoschek V, Keogh JS (2006) Molecular phylogeny of sea snakes reveals a rapidly diverged adaptive radiation. Biol J Linn Soc 89:523-539

Lukoschek V, Waycott M, Dunshea G (2005) Isolation and characterization of microsatellite loci from the Australasian sea snake, Aipysurus laevis. Mol Ecol Notes 5:875-881

Lukoschek V, Waycott M, Keogh JS (2008) Relative information content of polymorphic microsatellites and mitochondrial DNA for inferring dispersal and population genetic structure in the olive sea snake, Aipysurus laevis. Mol Ecol 17:3062-3077

Mackiewicz M, Tatarenkov A, Perry A, Martin R, Elder JFJ, Bechler DL, Avise JC (2006) Microsatellite documentation of malemediated outcrossing between inbred laboratory strains of the self-fertilizing mangrove killifish (Kryptolebias marmoratus). J Hered 97:508-513

Peakall R, Smouse PE (2006) genalEx 6: genetic analysis in Excel. Population genetic software for teaching and research. Mol Ecol Notes 6:288-295

Raymond M, Rousset F (1995) GENEPOP (version 1.2): population genetics software for exact tests and ecumenicism. J Hered 86: 248-249

Rousset F (2008) Genepop'007: a complete reimplementation of the Genepop software for Windows and Linux. Mol Ecol Res 8:103-106 